\title{
Quinacrine Fluorescent Karyotypic Pattern in Pennisetum americanum (L.) Leeke
}

\author{
K. A. A. J. Prabakaran, P. Vaidyanathan \\ and S. R. Sree Rangasamy \\ School of Genetics, Tamil Nadu Agricultural University, \\ Coimbatore-641 003, India
}

Accepted November 16, 1990

The introduction of the fluorescent dye, quinacrine, has been a major advance in cytogenetics in recent years, revolutioning research methods and providing a quick and reliable tool for karyotype analysis. Vosa (1971) demonstrated for the first time the quinacrine banding in plants. Later this method was extended to many plant species like Petunia hybrida (Smith and Oud, 1972), Rhoeo discolor (Natarajan and Natarajan, 1972), Scilla siberica (Vosa, 1973), Crepis capillaris (Schweizer, 1973), Allium (Vosa, 1976), Paris polyphylla (Filion and Vosa, 1980; Smith and Ingram, 1986). The karyotype of Pennisetum americanum has been studied very extensively by many workers (Virmani and Gill, 1972; Tyagi, 1975; Jauhar 1981). In the present investigation an attempt was made to study the $Q$ banding pattern in pearl millet.

Materials and methods

\section{Preparation of airdried slides}

Seeds of Pearl millet (UCH 11) were sown on wet filter paper in petri dishes and roots of 10-20 mm length were excised and pretreated with 0.01 per cent colchicine for $30-40 \mathrm{~min}$. at room temperature. Pretreated roots were washed free of colchicine with distilled water and fixed in 1:3 aceticalcohol for $24 \mathrm{hrs}$. at $4^{\circ} \mathrm{C}$.

\section{Maceration and squashing}

Maceration was done in 45 per cent acetic acid at $60^{\circ} \mathrm{C}$ for $15-30 \mathrm{~min}$. or placed in 9 parts of 45 per cent acetic acid +1 part of $\mathrm{NHCl}$ in a watch glass, warmed gently over flame until the solution steamed and left in solution for $1 \mathrm{~min}$. The root tips were washed in deionised water for 10-30 $\mathrm{min}$. at room temperature.

Enzymatic maceration using 2 per cent pectinase and 1 per cent cellulase in 5 per cent manitol was tried to get good chromosome spreads by dissolving cell wall. The meristematic region of the macerated root tip was placed in a drop of 45 per cent acetic acid on a well cleaned glass slide (or abluminized glass slide), a cover glass placed on it tapped gently and squashed strongly.

\section{Air drying}

The cover slips were flipped off from the glass slide by quick-freeze method (Conger and Fairchild, 1953). The slides were frozen on a block of dry ice or liquid nitrogen for $30 \mathrm{sec}$. and the coverslips were lifted from one corner with a razor blade. The slides and coverslips were transferred to 95 per cent alcohol and absolute alcohol for $5 \mathrm{~min}$. each before thawing and air dried. 


\section{Quinacrine staining}

The methods outlined by Vosa $(1970,1971)$, Vosa and Marchi (1972) and Darlington and Lacour (1974) were adopted. The dry slides were stained with 0.5 per cent Quinacrine dihydrochloride (Atebrin) in water (or) alcohol for 5-20 min. The stained slides were rinsed briefly 2-3 times in distilled water or alcohol according to whether aqueous or alcoholic solutions of the stain used. The slides were mounted in water or buffer solution (pH 6.8) and ringed with rubber solution to prevent drying. The slides were viewed through Zeiss Ultraphot microscope using HBO 50 high pressure mercury vapour lamp as a source of ultraviolet light and a $500 \mathrm{~nm}$ barrier filter and photomicrographs were taken using Konica 400 ASA colour $35 \mathrm{~mm}$ film.

\section{Identification of chromosomes}

The ' $Q$ ' banded chromosomes were arranged for Karyotypic analysis. The chromosomes were paired and numbered sequentially from 1 to 7 according to the length measurements made on chromosomes conventionally stained after ' $Q$ ' banding. The initial classification of chromosome was made based on length and centromeric position used for nonbanded chromosomes. Some mitotic cells showed considerable nonuniformity, in that homologous chromosomes differed greatly in overall fluorescent intensity and relative length. Identification was therefore based on the individual chromosomes rather than on its overall intensity. However, intensity was taken to serve as a secondary criterian if due allowance was made for non-uniformity.

\section{Results}

The $\mathrm{Q}$ banded karyotype and the $\mathrm{Q}$ banded chromosomes at different stages of division are presented (Fig. 1). An idiogram for the $\mathrm{Q}$ banded karyotype is depicted in Fig. 2.

At interphase, the chromosomes showed dots of high intensity fluorescence. The number and structure of the chromocentres in the interphase nuclei of Pennisetum americanum was variable according to the DNA content of the nuclei. Quinacrine staining at prophase stage showed well condensed chromocentres fluorescent more intensively than the other segments. The chromosomes at late prophase stage were stained intensively at specific regions and did not stain the other regions showing dull fluorescence. The chromosomes at prometaphase showed intense fluorescent and dull fluorescent regions but the clear cut region of the bands could not be identified. But, at metaphase stage all 14 chromosomes were visible individually showing banded regions.

A reconstruction of the chromosome complement is shown in Fig. 2. Chromosomes 1, 3,4 and 6 showed polymorphism. In the first pair, one of the two homologous chromosomes showed an extra terminal band in the long arm showing polymorphism. The homologous chromosomes in the second pair showed similar banding patterns. They had only one terminal band of same length in the short arm. The Q-banding pattern of the third pair showed polymorphism and the two homologous chromosomes had dissimilar size of terminal bands in the short arm. The fourth pair was stained intensively near the centromeric region. One of the two homologous chromosomes had a small centromeric band when compared to the other and an extra terminal band showing polymorphism. The fifth pair had only a small telomeric band in the short arm similar in size in both homologous chromosomes. The sixth pair showed two telomeric bands in each chromosome and one telomeric band in the short arm was dissimilar in size in the two chromosomes. The seventh pair bearing NOR was banded in both telomeric ends showing similar banding pattern. Thus, a karyotype showing polymorphic character for the chromosome pairs 1, 3, 4 and 6 was obtained using $Q$ banding. 


\section{Discussion}

The observed number and structure of chromocentres were variable according to the DNA content of the nuclei. The chromosomes at prophase stage showed striking pattern of fluorescence produced by differential binding of fluorescent dye. Conventional staining with Giemsa after Quinacrine staining showed that the intense fluorescent bands correspond for the most part with the condensed segments at prophase. This evidently demonstrates characteristic differences between the structure of DNA in eu- and heterochromatin. The condensed heterochromatin replicates the DNA later than euchromatin (Vosa, 1971). Hence, there appears a

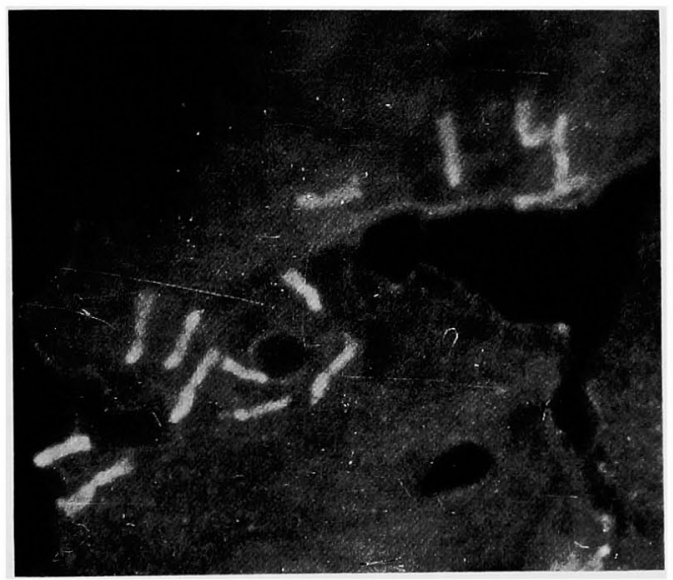

Fig. 1. Quinacrine stained chromosomes of Pearl millet.

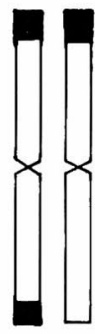

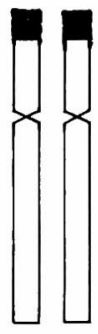

2

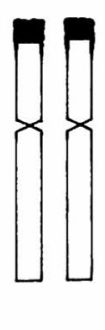

3

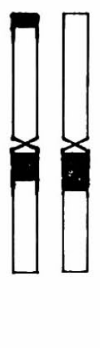

4

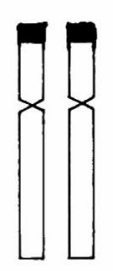

5

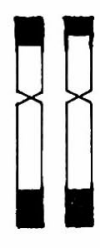

6

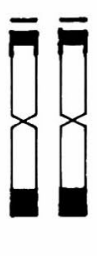

7

Fig. 2. Diagramatic representation of somatic chromosomes of Pennisetum americanum stained with Quinacrine.

good correlation between late replicating DNA, heterochromatin and intense fluorescent pattern. Various studies showed that quinacrine binds to DNA by intercalation between base pairs organisation of DNA and its association with nuclear proteins seem to affect the extent of such intercalation. So the $\mathrm{Q}$ bands resulting from differential excitation and quenching of the dye are influenced by the DNA base composition, chromosomal non histone proteins and also the type of binding to DNA (Babu and Verma, 1987).

The reconstructed chromosome complement showed chromosome polymorphism in the chromosome 1, 3, 4 and 6 . The homologues of these chromosomes differed in having bands of unequal size of different band positions. The chromosome polymorphism was also observed in Allium (Vosa, 1971, 1973). The chromosomes of peral millet showed Q bands only 
in telomeric and centromeric regions and intercalary bands were absent. Hence, using this technique, it is possible to identify individual chromosomes and even chromosomes with the same morphology can be differentiated using the banding patterns.

\begin{abstract}
The Q-banded karyotype of pearl millet was successfully demonstrated. The banding patterns at different stages were analysed and polymorphism in banding pattern of homologues of chromosomes 1, 3, 4 and 6 was observed. They differed in having bands of unequal size or different band positions. The chromosomes showed Q-bands only in telomeric and centromeric regions and intercalary bands were absent.
\end{abstract}

\title{
References
}

Babu, A. and R. S. Verma. 1987. Chromosome structure: Euchromatin and heterochromatin. Int. Rev. Cytol., 108: $1-60$.

Conger, A. D. and L. M. Fairchild. 1953. A quick freeze method for making smear slides permanent. Stain Technol., 28: 281-283.

Darlington, C. D. and L. F. LaCour. 1974. The handling of chromosomes. George Allen and Unwin Ltd., London. p. 272.

Filion, G. W. and C. G. Vosa. 1980. Quinacrine fluorescence studies in Paris polyphylla. Can. J. Genet. Cylol, 22: $417-420$.

Jauhar, P. 1981. Cytogenetics of pearl millet. Adv. Agron., 34: 407-479.

Natarajan, A. T. and S. Natarajan. 1972. The heterochromatin of Rhoeo discolor. Hereditas, 72: 323-330.

Schweizer, D. 1973. Differential staining of plant chromosome. Chromosoma, 40: 307-320.

Smith, M. C. and R. Ingram. 1986. Heterochromatin banding in the genus Paris. Genetica, 71: 141-145.

Smith, F. J. and J. C. Oud. 1972. The possibility of distinguishing chromosomes of Petunia hybrida by Quinacrine fluorescence. Genetica, 43: 589-596.

Tyagi, B. R. 1975. Karyomorphology of somatic chromosomes in pearl millet. Proc. Indian. Natl. Sci. Acad. 41 Part B; 462-465.

Virmani, S. S. and B. S. Gill. 1972. Somatic chromosomes of Pennisetum typhoides (Burm.) S \& H. Cytologia, 37: $257-260$.

Vosa, C. G. 1970. Heterochromatin recognition with fluorochromes. Chromosoma, 30: 366-372.

- 1971. The Quinacrine-Fluorescence patterns of the chromosomes of Allium carinatum. Chromosoma, 33: 382-385.

- 1973. The enhanced and reduced fluorescence bands and their relationship with the giemsa patterns in Allium flavum. Nobel Symposium, 23: 156-158.

- 1976. Heterochromatin patterns in Allium. I The relationship between the species of the cepa group and its allies. Heredity, 36: 383-392.

- and P. Marchi. 1972. On the quinacrine and giemsa patterns of the chromsomes of Vicia faba. Gior. Ital., 106: 151-159. 